\title{
The General Form-Invariance Principle
}

\author{
V. V. Voytik* \\ September 16, 2018
}

\begin{abstract}
Bashkir State Pedagogical University, ul. Oktyabr'skoi revoliutsii, 3a, Ufa, 450077, Russia Received December 15, 2010; in final form March 7, 2011; Published in the journal Gravitation and Cosmology, 2011, Vol. 17, No. 3, pp. 218-223
\end{abstract}

\begin{abstract}
We postulate the applicability of the general form-invariance principle in special relativity. It is shown that this principle holds in classical mechanics. Some examples of transformations between the reference frames which satisfy this principle are considered. A new transformation is proposed for a transition from a uniformly rotating reference frame to a reference frame whose origin is shifted from the rotation axis. Another possible formulation of this principle is given for the case of stationary rigid reference frames which differ from one another only by the position of the origin.
\end{abstract}

Key words: general principle of relativity, generalized principle of relativity, principle of general forminvariance, rigid frame reference, forminvariance of metric, generalized Lorentz transformation, shift transformation

PACS: 03.30. $+\mathrm{P}$

\section{Introduction}

The content of special relativity (SR) is to a greatest extent determined by the relativity principle. Therefore its different formulations are very important. The Poincaré-Einstein special relativity principle, corresponding exclusively to inertial reference frames, underwent a certain evolution from

*voytik1@yandex.ru 
its formulation in 1904 to the time when it became a general principle. Historically, the general relativity principle is closely related to the equivalence principle and the general covariance condition. At first there was no clear distinction between these notions. The first clear formulation of the general relativity principle as an independent statement that the laws of nature should be formulated in such a way that they hold with respect to arbitrarily moving coordinate systems has appeared only in 1915 in the paper "Relativity Theory". However, then and later the author saw the value of such an assumption in the equivalence principle (EP), i.e., in the fact that it enables one to replace a homogeneous field of gravity with a uniformly accelerated reference frame. The general covariance condition became a mathematical expression of this requirement.

The final formulation of the general relativity principle appeared in the work of 1916 "Foundations of General Relativity" [1] and did not substantially change afterwards. According to this principle, the equations that express the laws of nature must be generally covariant with respect to all continuous coordinate transformations. The general laws of nature should be expressed through equations valid in all coordinate systems, i.e. these equations should be covariant with respect to anty substitutions (generally covariant).

It is necessary to mention that there is at present [2] another, more general formulation of the special relativity principle, which is slightly weaker than Einstein's general relativity principle. It has received the name of the generalized relativity principle, which declares that "whatever physical reference frame we choose (inertial or non-inertial), one can always point out an infinite set of other reference frames such that all physical phenomena proceed in the same way with the initial reference frame; so that we do not have and cannot have any experimental opportunity to find out in which particular reference frame from this infinite set we are; for any non-inertial reference frames, there is an infinite set of other non-inertial frames in which all laws of physics are absolutely the same as in the initial system.

The main purpose of the present paper is as follows.

Above all, it is necessary to pay attention to the circumstance that the general relativity principle as it was initially formulated in 1915 has a certain value irrespective of the opportunity of replacing the field of inertia forces in a non-inertial reference frame with a gravitational field. The principle is applicable to both classical and relativistic phenomena and is one of the fundamentals of SR in non-inertial reference frames. Further on, in Section 2 we will give another, more rigorous formulation, and in Section 3 we will show that this principle originates from classical mechanics.

Moreover, if this principle holds, it is necessary to give examples of transformations between non-inertial reference frames that satisfy this principle. 
Such examples are considered in Section 4. In particular, we suggest a transformation of displacement from a uniformly rotating reference frame to a finite distance, which is in a certain sense similar to the Lorentz transformations.

Finally, in Section [5, another possible formulation of the general forminvariance principle will be given, specially for the case of stationary rigid reference frames which differ from each other only by the position of the origin.

\section{The general form-invariance principle for rigid reference frames}

The general relativity principle actually states that if one takes into account in some way the non-inertial motion of two different reference frames, then the mathematical form of the physical laws will remain invariable at transition from one frame to the other. The question is, how to do it in practice. The arbitrariness of the space-time metric in a given reference frame can be restricted by taking into account that it depends only on the internal kinematic characteristics of the reference body. The reference body (and hence the reference frame) is entirely characterized by only two vectors: the proper acceleration $\mathbf{W}$ and the proper angular velocity $\Omega$, and these parameters do not include the coordinates of the reference body, or its velocity, or any other a priori possible quantities. All distinctions between reference frames are connected exclusively with differences in these characteristics. Thus there is no way to distinguish one non-inertial reference frame from another if their origins have the same kinematic characteristics. This implies the non-existence of a unique, absolute reference frame, the only one with respect to which hold the laws of physics.

It can be therefore concluded that if one chooses another reference frame, different from the initial one by the most general transformation related to a translational motion along with rotation, the dependence of the space-time metric on the Cartesian coordinates will remain unchanged. It means that the physical laws, as the reference frame is changed, can only change due to changed vector characteristics of the reference frame, $\mathbf{W}$ and $\boldsymbol{\Omega}$, and the absolute values of the coordinates and velocity of the reference body are insignificant:

$$
g_{i k}(\mathbf{W}(T), \boldsymbol{\Omega}(T), \mathbf{R})=\text { forminvariant }
$$

We will call this statement the general form-invariance principle.

The condition that the space is Euclidean in an accelerated reference 
frame and the condition that the space-time is not curved uniquely fix the form of the metric in an arbitrary reference frame [3], 44] (here and henceforth $c=1)$ :

$$
\begin{gathered}
g_{00}=(1+\mathbf{W R})^{2}-(\boldsymbol{\Omega} \times \mathbf{R})^{2} \\
g_{0 \alpha}=-e_{\alpha \beta \gamma} \Omega_{\beta} R_{\gamma} \\
g_{\alpha \beta}=-\delta_{\alpha \beta}
\end{gathered}
$$

Mathematically, the general form-invariance principle means that in an arbitrary reference frame there exists such a transformation $X^{i}=X^{i}\left(x^{j}\right)$, or, in the three-dimensional form,

$$
\mathbf{R}=\mathbf{R}(\mathbf{r}, t), T=T(\mathbf{r}, t)
$$

that its substitution into the metric tensor transformation law does not change the form of the metric:

$$
\begin{gathered}
\frac{\partial X^{j}}{\partial x^{i}} \frac{\partial X^{l}}{\partial x^{k}} g_{j l}(\mathbf{W}, \boldsymbol{\Omega}, \mathbf{R})=g_{i k}(\mathbf{w}, \boldsymbol{\omega}, \mathbf{r}) \\
d s^{2}=\left\{(1+\mathbf{W R})^{2}-(\boldsymbol{\Omega} \times \mathbf{R})^{2}\right\} d T^{2}-2(\boldsymbol{\Omega} \times \mathbf{R}) d \mathbf{R} d T-d \mathbf{R}^{2}= \\
=\left\{(1+\mathbf{w r})^{2}-(\boldsymbol{\omega} \times \mathbf{r})^{2}\right\} d t^{2}-2(\boldsymbol{\omega} \times \mathbf{r}) d \mathbf{r} d t-d \mathbf{r}^{2}
\end{gathered}
$$

Such a transformation will be a change in the reference frame. The forminvariance of the metric guarantees that the physical laws will remain invariable as the reference frame is changed. These transformations evidently form a group.

Besides translational motion and rotations, a reference frame changes at displacements by a fixed distance. If the initial reference frame is rigid and non-stationary (i.e., its characteristics are time-dependent, $\mathbf{W}=\mathbf{W}(T)$, $\boldsymbol{\Omega}=\boldsymbol{\Omega}(T)$ ), then the reference frame whose origin is shifted will not be rigid, and its metric will not have the form (2)-(4). In this case, the question about the metric corresponding to such a reference frame requires an additional study. However, if the initial reference frame is rigid and stationary, the one with a shifted origin will also be rigid and stationary. For such a frame, (1) -(7) will be entirely applicable as well as all conclusions of this section.

The statement (66), (7) is necessary for any physical theory. This requirement is more restrictive than that of covariance at coordinate transformations, and it substantially restricts the class of possible transformations between reference frames to only those which leave invariable the functional dependence of the 4-dimensional metric on the coordinates of the instantaneously comoving inertial reference frame. The values of the kinematic parameters themselves can change. 
Maybe this condition does not deserve the name of a "principle" which is too obliging. But the same obvious nature is also inherent to the special relativity principle. But, as a matter of fact, the general form-invariance principle is a stronger statement than the generalized relativity principle. Indeed, if the vector parameters do not change as the reference frame is changing, then, accordingly, the numerical values and the functional form of the metric tensor at a given point of space do not change. Thus any physical phenomenon in the old and new reference frames, at the same initial conditions, will occur in the same way, so that ir will be impossible to distinguish one reference frame from another. It is this statement that is the essence of the generalized relativity principle. If the above parameters are zero, the generalized relativity principle passes on to the special relativity principle.

Let us stress that the general form-invariance principle is of great importance for SR. Indeed, there are no other reliable statements that would indicate the correctness of some transformation connecting two reference frames. Only in the case where the coordinate and time transformations keep the general form (7) it can be surely asserted that such a transformation between reference frames is truly related to a physical motion or relative dispositions.

\section{Validity of the general form-invariance prin- ciple for classical mechanics}

Let us now prove that the general form-invariance principle is valid for classical mechanics. To do that, it is sufficient to consider, in a non-inertial frame with the parameters $\mathbf{W}$ and $\Omega$, the simplest mechanical system, a free material point. It is convenient to use its Lagrangian in the form [5]

$$
L=\frac{m U^{2}}{2}+m \boldsymbol{\Omega}(\mathbf{R} \times \mathbf{U})+\frac{m(\boldsymbol{\Omega} \times \mathbf{R})^{2}}{2}-m \mathbf{W R}
$$

The most general transformation of the reference frame is a combination of motion with a displacement, a rotation and a turn of the axes. Let us first carry out a transformation of motion, i.e., a transition to the reference frame $s$ moving to a distance $\mathbf{b}(t)$ with the velocity $\mathbf{v}=d \mathbf{b} / d t$. Then the coordinates $\mathbf{R}$ and $\mathbf{r}$ and the velocities $\mathbf{U}$ and $\mathbf{u}$ in the systems $S$ and $s$ are related by

$$
\mathbf{R}=\mathbf{r}+\mathbf{b}, \mathbf{U}=\mathbf{u}+\mathbf{v}
$$

Substituting (9) into (8) and excluding full time derivatives, we obtain a new Lagrangian in the form 


$$
\begin{gathered}
L^{\prime}=\frac{m u^{2}}{2}+m \boldsymbol{\Omega}(\mathbf{r} \times \mathbf{u})+\frac{m}{2}(\boldsymbol{\Omega} \times \mathbf{r})^{2}- \\
-m\left[\mathbf{W}+\frac{d \mathbf{v}}{d t}+\boldsymbol{\Omega} \times \mathbf{v}+\boldsymbol{\Omega} \times(\boldsymbol{\Omega} \times \mathbf{b})+\frac{d(\boldsymbol{\Omega} \times \mathbf{b})}{d t}\right] \mathbf{r}
\end{gathered}
$$

The Lagrangian (10) has the form (8). The characteristics of the new reference frame are

$$
\boldsymbol{\omega}=\Omega, \mathbf{w}=\mathbf{W}+\dot{\mathbf{v}}+\dot{\Omega} \times \mathbf{b}+2 \Omega \times \mathbf{v}+\Omega \times(\Omega \times \mathbf{b}) .
$$

Thus automatically, from the validity of the general form-invariance condition, we have obtained the transformation laws for the characteristics of a reference frame at its change. From (11) it is evident that the choice of another reference body, irrespective of the state of its motion (it can even be at rest with respect to the first one) leads in general to a change of the reference frame since its vector characteristics change.

Let us now pass on to a reference frame $s$ rotating with respect to $S$ with an angular velocity $\boldsymbol{\nu}$ around a common center. Then the coordinates $\mathbf{R}$ and $\mathbf{r}$ in the frames $S$ and $s$ are related by the equations

$$
R_{\alpha}=a_{\alpha \beta}(t) r_{\beta}
$$

The rotation matrix $a_{\alpha \beta}$ satisfies the equalities

$$
a_{\alpha \beta} a_{\alpha \gamma}=\delta_{\beta \gamma}, \frac{d a_{\alpha \beta}}{d t}=e_{\beta \mu \lambda} \nu_{\lambda} a_{\alpha \mu}
$$

where $\nu_{\alpha}$ are components of the angular velocity of the system $s$ in the moving coordinate system. Using a specific expression for the rotation matrix, e.g., in terms of the Euler angles, it is easy to verify the following equalities for rotation matrices, which, due to their form may be called the "annihilation equalities":

$$
e_{\alpha \mu \lambda} a_{\mu \beta} a_{\lambda \gamma}=e_{\mu \beta \gamma} a_{\alpha \mu}, e_{\alpha \mu \lambda} a_{\beta \mu} a_{\gamma \lambda}=e_{\mu \beta \gamma} a_{\mu \alpha}
$$

Differentiating (12), we obtain that the velocity $\mathbf{U}$ is equal to

$$
U_{\alpha}=a_{\alpha \beta} u_{\beta}+e_{\beta \mu \lambda} \nu_{\lambda} a_{\alpha \mu} r_{\beta}
$$

Substituting (12) and (15) into (8) and using the relations (13) and (14), we obtain a new Lagrangian of the same form as (8) where the characteristics of the new reference frame are

$$
w_{\alpha}=a_{\beta \alpha} W_{\beta}, \omega_{\alpha}=a_{\beta \alpha} \Omega_{\beta}+\nu_{\alpha} .
$$

Thus the validity of the general form-invariance principle for classical mechanics has been proved. 


\section{Examples of transformations satisfying the general form-invariance principle}

As an example of using this principle, let us find the transformation of displacement by a vector $\mathbf{b}$ in an accelerated reference frame $(\boldsymbol{\Omega}=0)$. Let us assume that it is a usual substitution of the form $\mathbf{R}=\mathbf{r}+\mathbf{b}$. Inserting it in (7) and making obvious transformations, one can reduce the interval to the form

$$
d s^{2}=\left(1+\frac{\mathbf{W}}{1+\mathbf{W} \mathbf{b}} \mathbf{r}\right)^{2} d\left(T+\mathbf{b} \int_{0}^{T} \mathbf{W} d T\right)^{2}-d \mathbf{r}^{2}
$$

The metric found from here is a special case of (2)-(44), hence the assumption we made is true. In passing, we have obtained here the transformation of time

$$
t=T+\mathbf{b} \int_{0}^{T} \mathbf{W} d T
$$

From (17) it is evident that a displacement by a vector $\mathbf{b}$ within an accelerated reference frame changes the proper acceleration of the system to

$$
\mathbf{w}=\frac{\mathbf{W}}{1+\mathbf{W b}}
$$

This formula has been known for a long time [6].

Another example of a coordinate and time transformation (5) is the Lorentz-Nelson transformation of motion (it is the so-called generalized Lorentz transformation) which transforms an inertial reference frame $S(T, \mathbf{R})$ into an accelerating and rotating one, $s(t, \mathbf{r})$ :

$$
\begin{gathered}
T=\frac{\mathbf{v r}}{\sqrt{1-v^{2}}}+\int_{0}^{t} \frac{d t}{\sqrt{1-v^{2}}} \\
\mathbf{R}=\mathbf{r}+\frac{1-\sqrt{1-v^{2}}}{v^{2} \sqrt{1-v^{2}}}(\mathbf{v r}) \mathbf{v}+\int_{0}^{t} \frac{\mathbf{v} d t}{\sqrt{1-v^{2}}}
\end{gathered}
$$

That is, the interval $d s^{2}=d T^{2}-d \mathbf{R}^{2}$ reduces to the form (7) where $\mathbf{w}$ is

$$
\mathbf{w}=\frac{\dot{\mathbf{v}}}{\sqrt{1-v^{2}}}+\frac{1-\sqrt{1-v^{2}}}{v^{2}\left(1-v^{2}\right)}(\dot{\mathbf{v}} \mathbf{v}) \mathbf{v}
$$

while $\boldsymbol{\omega}$ is the Thomas precession frequency [7] (see also [8]):

$$
\boldsymbol{\omega}=\boldsymbol{\omega}_{T}=\frac{1-\sqrt{1-v^{2}}}{v^{2} \sqrt{1-v^{2}}} \mathbf{v} \times \dot{\mathbf{v}}
$$


where

$$
\dot{\mathbf{v}}=\frac{d \mathbf{v}}{d t}
$$

Lastly, one more illustration of a transformation between reference frames is the suggested transformation of displacement by a vector $\mathbf{b}(b, 0,0)$ with respect to an initial reference frame, uniformly rotating with an angular velocity $\Omega$ around the $Z$ axis in the Cartesian coordinate system $S(T, X, Y, Z)$ :

$$
\begin{gathered}
X=(b+x) \cos \frac{\Omega V y}{\sqrt{1-V^{2}}}+\frac{y}{\sqrt{1-V^{2}}} \sin \frac{\Omega V y}{\sqrt{1-V^{2}}} \\
Y=\frac{y}{\sqrt{1-V^{2}}} \cos \frac{\Omega V y}{\sqrt{1-V^{2}}}-(b+x) \sin \frac{\Omega V y}{\sqrt{1-V^{2}}} \\
Z=z \\
T=\frac{t+V y}{\sqrt{1-V^{2}}}
\end{gathered}
$$

where

$$
V=\Omega b
$$

If $V \ll 1, x \ll 1 / \Omega, y \ll 1 / \Omega$, the spatial transformation (24)-(26) passes over to the classical displacement transformation

$$
X=b+x, Y=y, Z=z
$$

Equation (27) has been found by H. Nikolic̀ [9]. At the transformation (24)(27), the proper acceleration $\mathbf{w}$ of the new reference frame $s(t, x, y, z)$ is

$$
w_{x}=-\frac{\Omega V}{1-V^{2}}, w_{y}=w_{z}=0
$$

and the new proper angular velocity $\boldsymbol{\omega}$ is

$$
\omega_{z}=\frac{\Omega}{1-V^{2}}, \omega_{x}=\omega_{y}=0
$$

This expression for the angular velocity is also known for a long time [10].

\section{The general form-invariance principle in its applications to stereometry and time}

There are such physical laws (e.g., the least truncated action principle, the Fermat principle) whose mathematical formulation contains, instead of the 
space-time metric tensor, the metric tensor of space in a non-inertial reference frame. In this connection, we will present another, equivalent formulation of the general form-invariance principle for stationary rigid reference frames, distinct from each other only by the position of their origins in the coordinate system of one of them.

Since the metric tensor remains form-invariant at displacements, also form-invariant must be the combination of these coefficients specifying the three-dimensional spatial metric and the "metric" of physical time. If the mathematical form of the spatial metric changed due to displacement, there would be a geometric way of choosing a certain absolute reference frame from the set of different stationary reference frames with the same characteristics, so that the spatial metric would be the simplest in terms of this absolute frame. This is certainly an incredible opportunity. In a similar way, the mathematical form of the element of physical time of a local inertial reference frame comoving to a given point also does not change, otherwise there would be a way of choosing an absolute reference frame using a clock.

Thus at a displacement from an arbitrary reference frame to another one, distinct from the first one by its constant position in the coordinate system of the first frame, the metrics of space and physical time in the new reference frame in the new Cartesian coordinate system do not change (are form-invariant). What will change are only the proper characteristics of the reference frame, whereas the numerical values and the mathematical form of the elements of distance and physical time should remain invariable. The standard definitions of the spatial metric and physical time

$$
\begin{gathered}
\gamma_{\alpha \beta}=-g_{\alpha \beta}+\frac{g_{0 \alpha} g_{0 \beta}}{g_{00}} \\
\delta \tau=\sqrt{g_{00}} d x^{0}+\frac{g_{0 \alpha}}{\sqrt{g_{00}}} d x^{\alpha}
\end{gathered}
$$

for an arbitrary stationary reference frame give general expressions for $d l^{2}$ and $\delta \tau$ having the form

$$
\begin{gathered}
d l^{2}=d \mathbf{R}^{2}+\frac{[(\boldsymbol{\Omega} \times \mathbf{R}) d \mathbf{R}]^{2}}{(1+\mathbf{W} \mathbf{R})^{2}-(\boldsymbol{\Omega} \times \mathbf{R})^{2}}=\text { form - invariant } \\
\delta \tau=\sqrt{(1+\mathbf{W} \mathbf{R})^{2}-(\boldsymbol{\Omega} \times \mathbf{R})^{2}} d T- \\
-\frac{(\boldsymbol{\Omega} \times \mathbf{R}) d \mathbf{R}}{\sqrt{(1+\mathbf{W R})^{2}-(\boldsymbol{\Omega} \times \mathbf{R})^{2}}}=\text { form }- \text { invariant }
\end{gathered}
$$

An example of using the general form-invariance principle in the form (34), (35) is the full displacement transformation from a uniformly rotating reference 
frame (24)-(27) presented above, and in the form (34) it gives a shortened transformation (i.e., only its spatial part (24)-(26) $)$. The proper characteristics of the new reference frame after the displacement can be obtained not only from the general form-invariance principle in the form (7) but also from invariance of the forms (34) and (35). For example, in a rotating reference frame $(\mathbf{W}=0)$ the length element is

$$
d l^{2}=d \mathbf{R}^{2}+\frac{[(\boldsymbol{\Omega} \times \mathbf{R}) d \mathbf{R}]^{2}}{1-(\boldsymbol{\Omega} \times \mathbf{R})^{2}}
$$

or

$$
d l^{2}=d X^{2}+d Y^{2}+d Z^{2}+\frac{\Omega^{2}(X d Y-Y d X)^{2}}{1-\Omega^{2}\left(X^{2}+Y^{2}\right)}
$$

At the transformation (24)-(26), the following equalities hold:

$$
\begin{gathered}
X^{2}+Y^{2}=(b+x)^{2}+\frac{y^{2}}{1-V^{2}} \\
X d Y-Y d X=\frac{[1-V(V+\Omega x)](b+x) d y}{\sqrt{1-V^{2}}}-\frac{\Omega V y^{2} d y}{\sqrt{1-V^{2}}}-\frac{y d x}{\sqrt{1-V^{2}}} \\
d X^{2}+d Y^{2}+d Z^{2}=d x^{2}+\frac{[1-V(V+\Omega x)]^{2} d y^{2}}{1-V^{2}}+\frac{\Omega^{2} V^{2} y^{2} d y^{2}}{\left(1-V^{2}\right)^{2}}+ \\
+\frac{2 \Omega V y d x d y}{1-V^{2}}+d z^{2}
\end{gathered}
$$

Substituting them into (37), we obtain

$$
\begin{aligned}
& d l^{2}=\left\{\frac{\Omega^{2} V^{2} x^{2} d y^{2}}{1-V^{2}}-2 V \Omega x d y^{2}-V^{2} d y^{2}+\frac{\Omega^{2} V^{2} y^{2} d y^{2}}{\left(1-V^{2}\right)^{2}}+\frac{2 \Omega V y d x d y}{1-V^{2}}\right\}+ \\
& +d x^{2}+d y^{2}+d z^{2}+\frac{\Omega^{2}\left([1-V(V+\Omega x)](b+x) d y-\frac{\Omega V y^{2} d y}{1-V^{2}}-y d x\right)^{2}}{\left(1-V^{2}\right)\left[1-(V+\Omega x)^{2}\right]-\Omega^{2} y^{2}}
\end{aligned}
$$

Inserting the term in the curly brackets in (41) to the numerator of the fraction, opening the brackets and collecting the terms, after rather bulky calculations we reduce the expression (41) to the form

$$
d l^{2}=d x^{2}+d y^{2}+d z^{2}+\frac{\Omega^{2}(x d y-y d x)^{2}}{(1-V(V+\Omega x))^{2}-\Omega^{2}\left(x^{2}+y^{2}\right)}
$$

The expression (42), written in a vector form, is equivalent to (34), where the quantities $\mathbf{w}$ and $\boldsymbol{\omega}$ are given by (30) and (31), respectively. 


\section{Conclusions}

The role of the general form-invariance principle in SR is that it is a kind of "selection rule" allowing for singling out from the whole set of transformations the true coordinate and time transformations corresponding to a transition from one reference frame to another. In cases where the coordinate transformations preserve the general forms like (22)-(44),(34), (35) , one can assert that such transformations between reference frames are not a trivial mathematical substitutions of spatial coordinates and time within the same reference frame but are connected with physical motion or relative positions. An important practical consequence of this principle are the Lorentz-Nelson motion transformation (20), (21), and the displacement transformation (24)-(27) from a rotating reference frame.

The hierarchy of principles of SR by their degree of generality is at present viewed as follows. A foundation of SR is the principle that the geometry is the Minkowski pseudo-Euclidean one. Its direct consequence is the general forminvariance principle, and then follow the generalized and special relativity principles. 


\section{References}

[1] A. Einstein, Die Grundlage der allgemeinen Relativitatstheorie, Ann. Physik 49, 769 (1916).

[2] A. A. Logunov, Lectures on Relativity and Gravitation: Modern Analysis of the Problem (Nauka, Moscow, 1987, in Russian).

[3] C. Møller. On Homogeneous Gravitational Fields in the General Theory of Relativity and the Clock Paradox, Trans. Dan. Acad. Sci. 2 (19), p.3, Kobenhavn, 1943.

[4] Ch. Misner, K. S. Thorne, and J. A. Wheeler, Gravitation, v. 1 (Freeman, San Francisco, 1970)

[5] L. D. Landau and E. M. Lifshitz, Mechanics (3rd ed., ButterworthHeinemann, 2003).

[6] C. Møller, Relativity Theory (2nd ed., Clarendon Press, Oxford, 1972).

[7] R. A. Nelson, Generalized Lorentz Transformation for an Accelerated, Rotating Frame of Reference, J. Math. Phys. 28, 2379 (1987).

[8] R. A. Nelson. Erratum: Generalized Lorentz Transformation for an Accelerated, Rotating Frame of Reference, J. Math. Phys. 35, 6224 (1994).

[9] H. Nikolic̀, Relativistic Contraction and Related Effects in Noninertial Frames", Phys. Rev. A 61, 032109 (2000).

[10] W. M. Irvine. "Electrodynamics in a Rotating System of Reference", Physica 30 (6), 1160 (1964). 